\title{
Very long-chain acyl-CoA dehydrogenase deficiency presenting as acute hypercapnic respiratory failure
}

\author{
M.K.H. Tong*, C-S. Lam*, T.W.L. Mak", M.Y.P. Fu*, S-H. Ng*, R.J.A. Wanders ${ }^{\star}$ and N.L.S. Tang ${ }^{+}$
}

ABSTRACT: Very long-chain acyl-CoA dehydrogenase deficiency (VLCAD) is a key enzyme catalysing the dehydrogenation of long-chain fatty acids in mitochondrial $\beta$-oxidation. VLCAD deficiency is a genetic disorder that commonly presents in infancy or childhood with episodes of hypoketotic hypoglycaemia, cardiomyopathy and liver dysfunction.

The present study reports an 18-yr-old Chinese female who presented with acute hypercapnic respiratory failure and rhabdomyolysis after a period of prolonged fasting and exertion. VLCAD deficiency was confirmed with decreased VLCAD activity in cultured fibroblasts.

The patient completely recovered with supportive care. Pulmonary function tests after the acute episode showed evidence of chronic subclinical respiratory muscle weakness.

In conclusion, this rare metabolic disorder should be considered in patients presenting with unexplained acute respiratory paralysis and failure.

KEYWORDS: Fatty acid oxidation, respiratory failure, rhabdomyolysis, very long-chain acyl-CoA dehydrogenase

$\mathbf{M}$ etabolic myopathies encompass a heterogeneous group of inherited disorders resulting in energy production defects in muscle tissues. Among these are disorders of fatty acid oxidation and glycogen storage diseases. Very long-chain acyl-CoA dehydrogenase (VLCAD) deficiency is a rare genetic enzymatic disorder in which the first and ratelimiting step in the $\beta$-oxidation of very long-chain fatty acids by the mitochondria is defective (fig. 1). There are three phenotypes of VLCAD deficiency, depending on the age and presentation, which can occur: 1) in the newborn with hypoketotic hypoglycaemia, liver dysfunction and cardiomyopathy; 2) later in infancy or childhood with episodes of hypoketotic hypoglycaemia and liver dysfunction; and 3) in adolescents or adults with exercise intolerance, recurrent myoglobulinaemia and rhabdomyolysis (table 1 ).

Here, the present authors report a case with dramatic presentation of rhabdomyolysis and acute muscle paralysis leading to respiratory failure in a Chinese female with previously undiagnosed VLCAD deficiency.

\section{CASE REPORT}

An apparently healthy 18 -yr-old female was admitted for generalised muscle pain and weakness for a duration of 1 day. The day before admission, she ate dinner at 16:00 $\mathrm{h}$ and had not eaten since then. The next morning, the patient woke up with generalised muscle pain and weakness, which worsened throughout the day.

She was finally brought to hospital that evening. By the time she was admitted to hospital, she had not eaten for $>24 \mathrm{~h}$.

The patient had a history of similar episodes of muscle pain and weakness, and passing dark urine since she was aged 10 yrs, but of lesser severity. These were usually brought on by moderate exercise. She denied the intake of any herbs or drugs and had no family history of similar conditions. On this occasion, the myalgia and weakness were more severe than previous episodes.

On examination, the patient was fully conscious but appeared weak. She was afebrile with a blood pressure of $126 / 77 \mathrm{mmHg}$, pulse 110 beats $\cdot \mathrm{min}^{-1}$,
AFFILIATIONS

Depts of *Medicine, and

\#Pathology, Tuen Mun Hospital,

Tuen Mun, and

+Dept of Chemical Pathology, The Chinese University of Hong Kong, Prince of Wales Hospital, Shatin, Hong Kong, China.

"Laboratory for Genetic Metabolic Diseases, Dept of Clinical Chemistry, Academic Medical Center, University of Amsterdam, Emma Children's Hospital, Amsterdam, The Netherlands.

CORRESPONDENCE

M.K.H. Tong

Dept of Medicine

Tuen Mun Hospital

Tuen Mun

Hong Kong

China

Fax: 85224569100

E-mail: khmtong@netvigator.com

Received:

November 252005

Accepted after revision: February 172006 


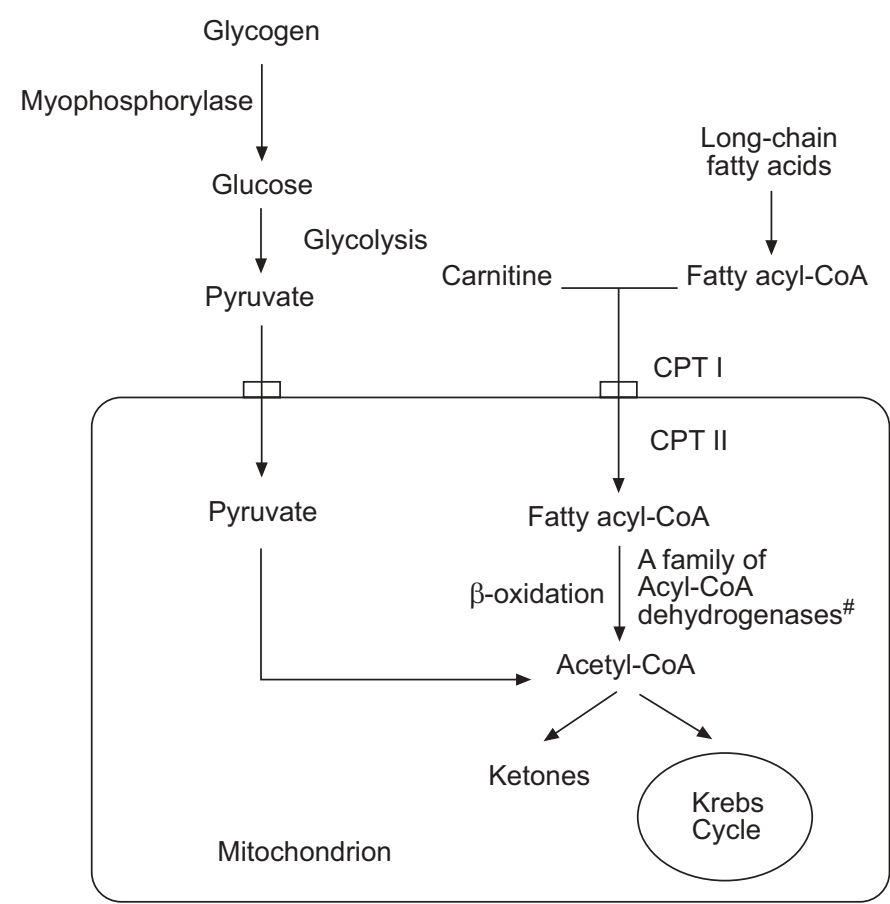

FIGURE 1. Fatty acid $\beta$-oxidation pathway. CPT: carnitine palmitoyltransferase. ${ }^{*}$ : reactions of $\beta$-oxidation are catalysed by acyl-CoA dehydrogenases specific for different chain lengths, including very long-chain, long-chain, medium-chain and short-chain acyl-CoA dehydrogenase.

respiratory rate 16 breaths $\cdot \mathrm{min}^{-1}$, and an arterial oxygen saturation of $96 \%$ in room air. Neurological examination revealed generalised muscle weakness of grade $4 / 5$, reflexes were diminished and limbs were hypotonic. Examinations of the respiratory and other systems were unremarkable.

Chest radiography showed a normal cardiac shadow and clear lung fields. Laboratory investigations revealed the following: white cell count $24.5 \times 10^{9} \cdot \mathrm{L}^{-1}$; sodium $133 \mathrm{mmol} \cdot \mathrm{L}^{-1}$; potassium $5.8 \mathrm{mmol} \cdot \mathrm{L}^{-1}$; creatine kinase $224,500 \mathrm{U} \cdot \mathrm{L}^{-1}$; lactate dehydrogenase $10,030 \mathrm{U} \cdot \mathrm{L}^{-1}$; and alanine transferase 909 $\mathrm{U} \cdot \mathrm{L}^{-1}$. Urinalysis was negative for ketones.

Within hours of admission, her muscle weakness worsened with the development of progressive respiratory paralysis and failure. Arterial blood gas measurement while the patient was on supplemental oxygen revealed the following: presence of respiratory acidosis and hypercapnia; $\mathrm{pH} 7.28$; arterial oxygen tension/inspiratory oxygen fraction ratio $56.76 \mathrm{kPa}$; carbon dioxide arterial tension $15.84 \mathrm{kPa}$; base excess $-4.5 \mathrm{mmol} \cdot \mathrm{L}^{-1}$; and bicarbonate $22.7 \mathrm{mmol} \cdot \mathrm{L}^{-1}$. She required endotracheal intubation and positive pressure ventilation. In the intensive care unit, serum urea and creatinine peaked at $6.1 \mathrm{mmol} \cdot \mathrm{L}^{-1}$ and $118 \mu \mathrm{mol} \cdot \mathrm{L}^{-1}$, respectively. The patient's condition stabilised and she was successfully weaned off the ventilator 10 days later.

A diagnosis of metabolic myopathy with rhabdomyolysis precipitated by fasting and exercise was suspected. Further investigations revealed the following: serum lactate $1.2 \mathrm{mmol} \cdot \mathrm{L}^{-1}\left(0.5-2.2 \mathrm{mmol} \cdot \mathrm{L}^{-1}\right)$; pyruvate $97 \mu \mathrm{mol} \cdot \mathrm{L}^{-1}(45-80$ $\left.\mu \mathrm{mol} \cdot \mathrm{L}^{-1}\right)$; and lactate/pyruvate ratio 12 (6-14). Muscle biopsy showed only nonspecific myositis changes; there were no ragged red fibres, inclusions or mitochondrial abnormalities and myofibrils were intact. The cytochrome oxidase and myophosphorylase staining were normal. Electron microscopy examination did not demonstrate abnormal organellar morphology. Urine organic acid profile showed a number of dicarboxylic acids, including adipic (C6) and suberic acids (C8). A $\beta$-oxidation defect was suspected. Serum free carnitine concentration and acylcarnitine pattern were determined by tandem mass spectrometry. The serum acylcarnitine profile found an increase in very long-chain acylcarnitines (C14:2 $0.26 \mu \mathrm{mol} \cdot \mathrm{L}^{-1}\left(<0.08 \mu \mathrm{mol} \cdot \mathrm{L}^{-1}\right) ; \mathrm{C} 14: 10.53 \mu \mathrm{mol} \cdot \mathrm{L}^{-1}(<0.18$ $\left.\left.\mu \mathrm{mol} \cdot \mathrm{L}^{-1}\right) ; \mathrm{C} 16: 10.16 \mu \mathrm{mol} \cdot \mathrm{L}^{-1}\left(<0.08 \mu \mathrm{mol} \cdot \mathrm{L}^{-1}\right)\right)$. The overall biochemical findings were highly suggestive of a VLCAD deficiency.

The diagnosis of VLCAD deficiency was confirmed by enzyme assays in cultured fibroblasts by $\left[{ }^{13} \mathrm{C}\right]$-palmitate loading test, which showed a marked reduction in VLCAD activity of

TABLE 1 Clinical manifestation and classification of very long-chain acyl-COA dehydrogenase (VLCAD) deficiency

\begin{tabular}{|c|c|c|c|c|c|}
\hline Phenotype & Onset & Symptoms and signs & Severity & Frequency $\%$ & Genotype \\
\hline Cardiomyopathic & Neonatal or early infancy & $\begin{array}{l}\text { Cardiomyopathy, pericardial } \\
\text { effusion, hepatic dysfunction, } \\
\text { mildly elevated serum ammonia, } \\
\text { lactate and creatine kinase levels }\end{array}$ & $\begin{array}{l}\text { Severe, usually fatal } \\
\text { without treatment }\end{array}$ & 47 & $\begin{array}{l}\text { Two null mutations with } \\
\text { no residual VLCAD } \\
\text { enzyme activity }\end{array}$ \\
\hline Hepatic & Late infancy or childhood & $\begin{array}{c}\text { Recurrent hypoketotic } \\
\text { hypoglycaemia, hepatic } \\
\text { dysfunction }\end{array}$ & $\begin{array}{l}\text { Intermediate, may be life } \\
\text { threatening if not } \\
\text { diagnosed and treated }\end{array}$ & 33 & $\downarrow$ \\
\hline Myopathic & Adolescence to adulthood & $\begin{array}{l}\text { Isolated skeletal muscle } \\
\text { involvement with episodic muscle } \\
\text { weakness, myalgia and } \\
\text { rhabdomyolysis triggered by } \\
\text { exercise or fasting }\end{array}$ & Mild & 20 & $\begin{array}{l}\text { Two missense mutations } \\
\text { or single amino acid } \\
\text { deletions with residual } \\
\text { enzyme activity }\end{array}$ \\
\hline
\end{tabular}

Arrow represents the shift from two null mutations through to two missense mutations. 
$0.8 \mathrm{nmol} \cdot \mathrm{min}^{-1} \cdot \mathrm{mg}^{-1}$ protein (normal range: $4.21 \pm 0.71 \mathrm{nmol}$. $\left.\mathrm{min}^{-1} \cdot \mathrm{mg}^{-1}\right)$, confirming a defect at the level of VLCAD.

The patient was discharged from hospital with full recovery and normal creatine kinase level after 27 days. Pulmonary function tests performed 3 months later, when the patient was asymptomatic, showed typical inspiratory and expiratory muscle weakness patterns (forced expiratory volume in one second $82 \%$ predicted, forced vital capacity $75 \%$, total lung capacity $60 \%$, residual volume $20 \%$, forced residual capacity $50 \%$, diffusing capacity of the lung for carbon monoxide $75 \%$, transfer coefficient of the lung for carbon monoxide $93 \%$, maximal minute ventilation $69 \%$, maximal expiratory pressure $28 \%$ and maximal inspiratory pressure $62 \%$ ).

\section{DISCUSSION}

Exercise intolerance and recurrent myoglobulinuria are the usual presenting features of the heterogeneous group of genetic muscle disorders with underlying deficiency in energy production arising from a wide variety of metabolic defects, including: defects in carbohydrate metabolism (glycolytic/ glycogenolytic disorders); fatty acid metabolism (deficiency of $\beta$-oxidation enzymes, carnitine deficiency syndromes and fatty acid transport defects); and electron transport chain pathways (mitochondrial defects; fig. 1). The clinical features of these metabolic myopathies may vary with the age of presentation. Defects of cellular energy production in the newborn and infants often give rise to severe multi-system disorders, whereas adult-onset disease is usually restricted to muscles.

For disorders of fatty acid metabolism, carnitine palmitoyltransferase (CPT) II deficiency is the most common defect, as well as being the most common metabolic cause of recurrent rhabdomyolysis. The clinical features in the patient were suggestive of a fatty acid metabolism disorder, but the subsequent biochemical assays indicated the presence of the rarer VLCAD deficiency instead.

VLCAD deficiency is a genetic disorder of mitochondrial fatty acid $\beta$-oxidation, resulting in a defect in oxidation of longchain fatty acids. After prolonged fasting, the body switches from carbohydrates to fatty acids for energy production. In VLCAD deficiency, because of the inability to utilise longchain fatty acids, prolonged fasting and excessive exertion predispose the patient to acute metabolic decompensation, hypoketotic hypoglycaemia, cardiomyopathy and rhabdomyolysis [1].

The enzyme VLCAD was first discovered by IZAI et al. [2] in 1992. The inheritance of this disorder is autosomal recessive. The exact frequency of the enzyme deficiency in the population is not known, but, in one pilot mass newborn screening programme the incidence of fatty acid oxidation defects, including short-chain, medium-chain, and very long-chain acyl-CoA dehydrogenase deficiencies, was found to be approximately one in 12,000 [3].

The phenotype of VLCAD deficiency is heterogeneous and may be clinically classified into three forms: 1) in the newborn with hypoketotic hypoglycaemia, liver dysfunction and cardiomyopathy; 2) later in infancy or childhood with episodes of hypoketotic hypoglycaemia and liver dysfunction; and 3) in adolescents or adults with recurrent rhabdomyolysis. Most patients have the severe childhood form, characterised by cardiomyopathy with early onset and poor outcome. In affected patients, as a result of the inability to metabolise fatty acids and generate ketone bodies, hypoketotic hypoglycaemia develops when glucose and glycogen stores are depleted. The patient in the present study belongs to the rare adult-onset type with rhabdomyolysis after exertion and without hypoglycaemia [4,5]. Affected individuals typically present with muscle weakness or passing dark-coloured urine after vigorous exercise. For the present patient, the common causes of rhabdomyolysis have been excluded, including muscle injury, sepsis, drugs, toxins and inflammatory myopathies. Muscle biopsy was nondiagnostic, showing nonspecific minor myopathic features, with normal cytochrome oxidase and myophosphorylase stainings, and no abnormal organellar morphology on electron microscopy. Fasting lactate was normal. Consequently, fatty acid metabolism disorder was suspected given the history of recurrent muscle pain (rhabdomyolysis) and dark urine (myoglobinuria) after prolonged exertion or fasting.

The initial diagnostic approach for metabolic myopathy in an acutely ill patient is the determination of the urinary dicarboxylic acids. The pattern in the patient was compatible with a fatty acid $\beta$-oxidation defect. The blood acylcarnitine pattern subsequently helps to differentiate the subtype of $\beta$ oxidation defect. In the present case, the serum and urine biochemical findings were highly suggestive of VLCAD deficiency. Enzymatic assays in cultured fibroblasts and genetic mutational analysis confirmed the diagnosis. The defective gene of VLCAD has been located to the short arm of chromosome 17 between bands P11.2 and P11.13105 [6]. The molecular basis of VLCAD deficiency is very diverse with $>80$ mutations reported in the literature $[7,8]$.

Muscle weakness to the degree of respiratory failure is uncommon in patients with rhabdomyolysis. It has been described in a few patients with CPT II deficiency, but respiratory failure has not been previously described in patients with VLCAD deficiency. This appears to be the first report of this disorder presenting as acute hypercapnic respiratory failure. The patient's late presentation to hospital might have accounted for its occurrence. The pulmonary function data obtained when the patient had fully recovered from the acute episode demonstrated typical findings of respiratory muscle weakness. This provides the first evidence of the presence of chronic subclinical respiratory muscle weakness in a patient with fatty acid metabolism defect. This case is also the first VLCAD deficiency reported in literature in a Chinese patient. With a high index of suspicion and supported by appropriate biochemical, enzymatic and mutational analysis, the current authors believe that more cases will be identified in the future.

In conclusion, genetic defects of fatty acid $\beta$-oxidation should be considered when an adolescent or adult patient presents with unexplained acute respiratory failure, muscle pain and rhabdomyolysis, which is induced by prolonged exercise and fasting.

\section{REFERENCES}

1 Aoyama T, Souri M, Ushikubo S, et al. Purification of human very-long-chain acyl-coenzyme A dehydrogenase 
and characterization of its deficiency in seven patients. J Clin Invest 1995; 95: 2465-2473.

2 Izai K, Uchida Y, Orii T, Yamamoto S, Hashimoto T. Novel fatty acid $\beta$-oxidation enzymes in rat liver mitochondria. I. Purification and properties of very-long-chain acyl-coenzyme A dehydrogenase. J Biol Chem 1992; 267: 1027-1033.

3 Naylor EW, Chace DH. Automated mass spectrometry for mass newborn screening for disorders in fatty acid, organic acid, and amino acid metabolism. J Child Neurol 1999; Suppl. 1, 14: S4-S8.

4 Ogilvie I, Pourfarzam M, Jackson S, Stockdale C, Bartlett K, Turnbull DM. Very long-chain acyl coenzyme A dehydrogenase deficiency presenting with exercise-induced myoglobinuria. Neurology 1994; 44: 467-473.
5 Smelt AH, Poorthuis BJ, Onkenhout W, et al. Very long chain acyl-coenzyme A dehydrogenase deficiency with adult onset. Ann Neurol 1998; 43: 540-544.

6 Wanders RJA, Vreken P, den Boer MEJ, Wijburg FA, van Gennip AH, IJlst L. Disorders of mitochondrial fatty acylCoA B-oxidation. J Inherit Metab Dis 1999; 22: 442-487.

7 Millington DS, Terada N, Chace $\mathrm{DH}$, et al. The role of tandem mass spectrometry in the diagnosis of fatty acid oxidation disorders. Prog Clin Biol Res 1992; 375: 339-354.

8 Orii KO, Aoyama T, Souri M, et al. Genomic DNA organization of human mitochondrial very-long-chain acylcoA dehydrogenase and mutation analysis. Biochem Biophys Res Commun 1995; 217: 987-982. 